\title{
Outcomes of liver transplantation using moderately steatotic liver from donation after cardiac death (DCD)
}

\author{
Xin Duan, Liting Yan, Yan Shen, Min Zhang, Xueli Bai, Tingbo Liang \\ Division of Hepatobiliary and Pancreatic Surgery, Department of Surgery, The First Affiliated Hospital, School of Medicine, Zhejiang University, \\ Hangzhou, China \\ Contributions: (I) Conception and design: X Duan; (II) Administrative support: T Liang; (III) Provision of study materials or patients: T Liang; (IV) \\ Collection and assembly of data: L Yan; (V) Data analysis and interpretation: Xin D; (VI) Manuscript writing: All authors; (VII) Final approval of \\ manuscript: All authors. \\ Correspondence to: Tingbo Liang. Division of Hepatobiliary and Pancreatic Surgery, Department of Surgery, The First Affiliated Hospital, School of \\ Medicine, Zhejiang University, 79 Qingchun Road, Hangzhou, China. Email: liangtingbo@zju.edu.cn.
}

Background: Liver grafts from donation after cardiac death (DCD) with moderate steatosis (MS) are generally considered unsui for liver transplantation (LT) because DCD and MS are independent risk factors of poor prognosis of LT. Many centers have begun to accept this type of liver graft. However, the clinical outcomes are indeed controversial. This study aimed to examine the outcomes after LT of using such liver grafts.

Methods: This study retrospectively reviewed our experiences in 80 allografts from May 2015 to September 2019. A total of 16 allografts using MS liver grafts from DCD (MS-DCD group) were compared with a matched control group of 64 allografts using a nonsteatotic liver graft (NS group) (1:4 ratio). Postoperative outcomes, including primary nonfunction (PNF), initial poor function (IPF), postoperative complications, and graft/patient survival rates, were extracted for pooled analysis.

Results: Recipient and surgical characteristics of patients and clinical data of donors between MS-DCD group and NS group were balanced. No significant differences were observed in hepatitis B virus (HBV) infection, model for end-stage liver disease (MELD) score, cold ischemia time (CIT), donor risk index, warm ischemia time (WIT). A significant difference was detected in the incidence of initial poor function (IPF) (11/16 vs. 26/64; $\mathrm{P}=0.02)$, and the average peak value of aspartate transaminase (AST) (3,469 vs. 1,295 ; $\mathrm{P}<0.01$ ) was significantly higher in the steatosis group. Meanwhile, alanine transaminase (ALT) was only higher on day 1, and international normalized ratio (INR) level was only higher on days 1 and 3 and disappeared on day 7. The serum total bilirubin (TB) was the same between the two groups. Postoperative complications were similar between the two groups. The 90-day, 1-year, and 3-year survival rates in patients and grafts between the two groups were similar (patient survival(MS-DCD group $v s$. NS group): $75 \% v s$. $85.9 \%, 75 \%$ vs. $78.1 \%, 68.8 \%$ vs. $71.9 \%$, log-rank test, $\mathrm{P}=0.77$; graft survival(MS-DCD group vs. NS group): $75 \%$ vs. $84.4 \%, 75 \%$ vs. $75 \%, 68.8 \%$ vs. $68.8 \%$, log-rank test, $\mathrm{P}=0.79)$.

Conclusions: After rigorous evaluation, it was found that moderately steatotic liver from DCD is an effective means to expand the source of liver supply.

Keywords: Liver transplantation; steatosis; donation after cardiac death (DCD)

Submitted Jun 30, 2020. Accepted for publication Sep 16, 2020.

doi: $10.21037 /$ atm-20-5888

View this article at: http://dx.doi.org/10.21037/atm-20-5888 


\section{Introduction}

Liver transplantation is the standard treatment for end-stage liver disease and is the only effective treatment for patients with acute liver failure and unresectable liver tumors. However, the pressure of liver graft shortages always exists. The gap between the number of patients needing transplants and the number of available organs is growing, and this situation forces clinicians to constantly try to expand the liver donor pool by utilizing expanded criteria donors (ECD). ECD includes steatotic donors, elderly or older donors, nonheart beating donors, donors with hypernatremia, donors with hypotension, donors with viral infections, donors with malignancies, donors with infections; however, all these donor factors are closely associated with poor prognosis after liver transplantation (1). Of these factors, steatosis and donation after cardiac death (DCD) are the most common sources of ECD (2), yet no consensus exists on how to safely use a liver graft from ECD.

The prevalence of obesity in the population has significantly increased the incidence of liver steatosis in the past decade. Some data indicate that the proportion of fatty liver in the population is $20-24 \%$ (3). This result has also been confirmed in donor livers (4). However, the use of steatosis donor livers is closely related to ischemiareperfusion injury and liver function decompensation after transplantation, with nearly one-third of donor livers being abandoned due to pathological fatty liver (5).

Factors of hepatic steatosis include obesity, advanced age, alcoholism, diabetes, hyperlipidemia, and changes in nutritional metabolism (5). Histologists divide liver steatosis into two categories. Of these, macrovesicular steatosis (MS) is more dangerous and involves fatty vacuoles occupying most of the hepatocyte cytoplasm and replacing the structure around the nucleus. The second category is microvesicular steatosis which involves small vacuoles and lobular distribution. Its pathological mechanism is believed to be related to mitochondrial damage and nutritional metabolic disorders; this change is largely reversible and does not tend to cause adverse consequences after liver transplantation. Studies have confirmed that hepatic microvesicular steatosis does not affect the final outcome of liver transplantation (6). MS, however, has been classified into three levels of severity: mild $(<30 \%)$, moderate $(30-$ $60 \%)$, and severe (>60\%). Mild steatosis donor livers $(<30 \%)$ can be safely used because the results after transplantation are similar to those of non-steered livers (7). Severe fatty degenerative donor livers $(>60 \%)$ are a significantly high-risk factor for liver failure after transplantation, and they should not be used (8). Although some centers have shared the experience of successful use of severe fatty liver donors, this success is only under the premise of strictly controlling the cold ischemia time (CIT $<8 \mathrm{~h}$ ) and the acquisition of donation after brain death (DBD). Meanwhile, the use of $30-60 \%$ moderate steatosis donor livers is rife with controversy (9). The incidence of liver failure after transplantation with moderate steatosis donors and (nonsteatosis) donor livers is reported to be $13 \%$ vs. $3 \%$, respectively (10).

DCD refers to patients with severe and irreversible brain injury or other diseases who fail to meet the standard of brain death, with the planned withdrawal of life support with the consent of family members and doctors, and then wait for the circulation stop). Currently, DCD donor livers are classified into two categories according to Maastricht classification (11): uncontrollable and controlled DCD. Uncontrollable DCD refers to cardiac arrest due to acute trauma. (Despite life resuscitation treatment, it is difficult to reverse death). Uncontrollable DCD usually occurs outside the hospital or emergency room and includes dead donors admitted to the hospital and those who die after resuscitation. Controlled DCD refers to cardiac arrest that occurs after planned withdrawal of life support and usually occurs in the intensive care unit (ICU) room or operating room, and include waiting for cardiac arrest donors and cardiac arrest with brain death donors. In the process of DCD donor donation, donor death is defined as irreversible cardiopulmonary cessation. Thus, the DCD donor liver must undergo a period of hypotension and hypoxia perfusion. The resulting warm ischemic injury is an important feature of a DCD donor liver. It is closely associated with the early onset of liver failure, arterial embolism, and biliary complications after recipient transplantation (12).

In global clinical practice, DCD has received extensive attention from the transplantation community and is considered a safe and effective way to expand the donor pool. In 2015, DCD accounted for approximately $17 \%$ of the total organ donation after the death of citizens worldwide (13). In China, the brain death law has not yet been implemented and the public's traditional perception of death is limited; thus, DCD organs may become an effective means for the Organ Procurement Organization (OPO) team to expand the organ pool as a major source of organ acquisition (13).

Given this, the choice of donor liver becomes particularly 
difficult when the double adverse factors of DCD and liver steatosis are superimposed.

In order to clarify the safety of using moderately steatotic liver from DCD, this study retrospectively analyzed and compared the clinical outcomes of MS-DCD donor liver and nonsteatosis donor liver, providing more favorable evidence for future clinical decision-making

We present the following article in accordance with the STROBE reporting checklist (available at http://dx.doi. org/10.21037/atm-20-5888).

\section{Methods}

The study was conducted in accordance with the Declaration of Helsinki (as revised in 2013). The study was approved by the First Affiliated Hospital, College of Medicine, Zhejiang University (No. 1393-1) and individual consent for this retrospective analysis was waived.

\section{Study population}

This study retrospectively analyzed 1104 dead donor liver transplants (excluding split liver transplantation, pediatric liver transplantation, retransplantation, and multi-organ combined transplantation) in our center from March 2015 to June 2019, during this time along with 807 patients with DCD liver (73\%), and 297 patients with DBD liver (27\%). According to the results of pathological biopsy after liver reperfusion, moderate steatosis was defined as $30-60 \%$ of MS, and the degree of microvesicular steatosis was not referenced. Among the 807 DCD liver patients, 16 patients received liver with moderate steatosis, which were used as the study group, and a random 1:4 ratio matching was performed to control for age, body mass index (BMI), model for end-stage liver disease (MELD), and CIT. A total of 64 patients who received liver with nonsteatosis were the control group. The postoperative follow-up data ended in September 2019, and the shortest follow-up time was 3 months.

\section{Preoperative procedures and surgical detail}

Donor liver procurement usually uses dual perfusion of the abdominal aorta and portal vein, kidney aorta or histidinetryptophan-ketoglutarate (HTK) solution for abdominal aortic perfusion, and University of Wisconsin (UW) solution for the portal vein. In this study, the donor liver was ultimately stored in UW solution. The amount of perfusion solution depended on the organ perfusion effect. When the color of the donor liver was uniform and the color of the perfusate was clear, the perfusion effect was considered good with $3,000-5,000 \mathrm{~mL}$ for the abdominal aorta and 2,000-3,000 $\mathrm{mL}$ for the portal vein. DCD liver procurement started 2 min after donor cardiac arrest, while DBD was acquired without waiting time.

Liver procurement was not performed by a fixed OPO team. Thus, all liver donors were evaluated by the center's liver transplant team before surgery. For donor livers that are yellow in color and have rounded edges, rapid frozen biopsies are necessary to provide information for the surgeon team. In general, the graft liver will be discarded when the DCD graft liver is $>60 \% \mathrm{MS}$ or liver fibrosclerosis lever II or more. After the donor's laboratory indicators, clinical characteristics, cold ischemia time (CIT)/warm ischemia time (WIT), and liver pathological results were comprehensively evaluated, it was finally decided whether the donor liver was to be used or discarded. In addition, our center used a modified piggyback liver transplantation. The anastomosis sequence included preferential anastomosis of the portal vein and inferior cavity, followed by anastomosis of the artery, and finally that of the bile duct.

\section{Pathological assessment}

After the donor liver perfusion was completed, the histopathology could be obtained. A $1 \times 1 \mathrm{~cm}$ sample of liver tissue was excised from the left and right liver margins and immediately fixed with $10 \%$ formalin solution. Then, hematoxylin-eosin staining was applied. The center's fulltime and experienced liver pathologists divided fatty donor livers into three categories according to the degree of MS: mild $(5-30 \%)$, moderate $(30-60 \%)$, and severe $(>60 \%)$. The rapid frozen biopsy was usually completed before the liver recipient surgery began. Besides steatosis, other pathological results were evaluated, including hepatocyte necrosis and fibrosis.

\section{Donor and recipient characteristics}

We collected donor data including age, gender, BMI, donor risk index (DRI, 3-25), WIT of DCD (time from donor removal of life support to aortic perfusion; WIT of DBD is recorded as 0 ), type and quantity of perfusion fluid, cause of death, and whether large doses of vasoactive drugs were used before donation. Recipient data included age, gender, primary disease, CIT, MELD score, mean operation time, 
intraoperative blood loss, blood transfusion volume (red blood cells), ICU stay, and hospital stay.

\section{Postoperative outcomes}

Postoperative outcome analysis included primary nonfunction (PNF), IPF, major complications after liver transplantation, graft survival, and patient survival. PNF was defined as irrecoverable graft failure that occurred within 7 days after transplant that led to retransplantation or patient death. IPF was defined as the peak serum values of aspartate aminotransferase/alanine aminotransferase (ALT/AST) $>1,500 \mathrm{mmol} / \mathrm{L}$ within 7 days after transplant. We also recorded the serum ALT/AST, bilirubin, and international normalized ratio (INR) levels within 7 days after surgery and analyzed the trend changes. The main complications after transplantation included arterial embolism, portal embolism, abdominal hemorrhage, acute renal failure, and acute rejection. Biliary complications, including bile leaks and biliary strictures (anatomical and non-anatomical stenosis), were counted separately. Organ survival referred to the time from the beginning of transplantation to the death or retransplantation of patients, and patient survival referred to the time from the beginning of transplantation to the death of patients.

\section{Statistical analysis}

Continuous variables are shown as the mean \pm standard deviation (SD) or medians with the 25th-75th interquartile ranges when presenting significant differences from the normal distribution. For continuous variables, either the Mann-Whitney U-test or Student's $t$-test was performed. Categorical variables were presented as numbers and percentages and compared using the Pearson's Chi-square test or the Fisher's exact test if appropriate. Patient and graft survival rates were analyzed according to the Kaplan-Meier method, and comparison of survival was performed using the log-rank test. Statistical significance was indicated by $\mathrm{P}$ values lower than 0.05 . Statistical analyses were performed using the statistical software package SPSS version 25.0 (SPSS Inc., Chicago, IL, USA).

\section{Results}

\section{Clinical characteristics}

Recipient and surgical characteristics of patients in the group with DCD steatosis and the nonsteatosis control group are summarized in Table 1. The basic characteristics and indications for transplant were similar. No significant differences were observed in the hepatitis $\mathrm{B}$ virus (HBV) infection (12/16 vs. 52/64; $\mathrm{P}=0.83)$, MELD score (13 vs. 18; $\mathrm{P}=0.18$ ), CIT (9.5 vs. $10.3 \mathrm{~h} ; \mathrm{P}=0.40$ ), or other factors between the two groups.

The clinical data of donors between the two groups are presented in Table 2. The basic characteristics were balanced. In the control group, we set the WIT, which started when the donor's life support was removed, as $0 \mathrm{~min}$ when the type of donor was DBD. The median of WIT was $15 \mathrm{~min}$ in this group, and the maximum was $68 \mathrm{~min}$. In the DCD steatosis group, the median and maximum of WIT were 16 and $35 \mathrm{~min}$, respectively. No significant difference was detected in WIT $(\mathrm{P}=0.78)$. No differences were found in DRI (1.2 vs. 1.2; $\mathrm{P}=0.54)$, WIT (16 vs. 15 minutes; $\mathrm{P}=0.78)$, HBV (0/16 vs. 6/64; $\mathrm{P}=0.46)$, or other risk factors.

\section{Postoperative outcomes and complications}

Operative outcomes of recipients in two groups are listed in Table 3. During the first week after transplantation, a significant difference was found in the incidence of IPF (11/16 vs. 26/64; $\mathrm{P}=0.02)$, while the incidence of $\mathrm{PNF}$ $(1 / 16$ vs. $5 / 64 ; \mathrm{P}>0.99)$ was similar in the two groups. In the control group, one patient received retransplantation and survived, while the others in the two groups died of PNF before retransplantation. For laboratory markers of liver function, the average peak value of transaminase AST $(3,469$ vs. 1,$295 ; \mathrm{P}<0.01)$ was significantly higher in the group with steatosis than in the control group during the week after transplantation. Meanwhile, ALT was only higher on day 1, and INR was only higher on days 1 and 3 and disappeared on day 7. The serum (TB) was the same between the two groups (Figures 1 and 2). The postoperative complications, such as portal thrombosis and biliary complications, and ICU stay were similar between the two groups. The median postoperative follow-up was 14 months (range, 0-58 months).

\section{Recipient survival}

The overall patient survival rates at 90 days, 1 year, and 3 years in patients with steatotic grafts and the controls were $75 \%$ versus $75 \%, 68.8 \%$ and $85.9 \%$, and $78.1 \%$ versus $71.9 \%$, respectively ( $\mathrm{P}=0.77$; Figure 3$)$. No difference was found between the two groups (Table 4). Similarly, we found 
Table 1 Recipient and surgical characteristics in patients

\begin{tabular}{|c|c|c|c|}
\hline Variable & DCD steatosis group $(n=16)$ & Control group $(\mathrm{n}=64)$ & $P$ value \\
\hline $\operatorname{Sex}(M / F)$ & $12 / 4$ & $50 / 14$ & $>0.99$ \\
\hline BMI $\left(\mathrm{kg} / \mathrm{m}^{2}\right)$ & 24.3 (19.0-25.9) & $22.8(20.7-25.2)$ & 0.91 \\
\hline \multicolumn{4}{|l|}{ Etiology } \\
\hline Acute liver failure & $6(37.5 \%)$ & $18(28.1 \%)$ & 0.67 \\
\hline Liver cancer & $4(25.0 \%)$ & $13(20.3 \%)$ & 0.95 \\
\hline HBV-related liver disease & $12(75.0 \%)$ & $52(82.3 \%)$ & 0.83 \\
\hline MELD score & $13(8.0-27.8)$ & $18(10-29)$ & 0.18 \\
\hline Blood loss (mL) & $1,000(525-1,875)$ & $1,000(600-1,750)$ & 0.93 \\
\hline Use of blood products & $11(68.8 \%)$ & $51(79.7 \%)$ & 0.55 \\
\hline RBC (units) & $4.75(0-7.1)$ & $6.0(3.5-8.9)$ & 0.35 \\
\hline FFP $(m L)$ & $1,055(965-1,527.5)$ & 1,195 (920-1,825) & 0.62 \\
\hline
\end{tabular}

DCD, donation after cardiac death; HBV, hepatitis B virus; BMI, body mass index; MELD, model for end-stage liver disease; CIT, cold ischemia time; RBC, red blood cell; FFP, fresh frozen plasma.

no significant differences in 90-day, 1-year, and 3-year graft survival rates between the two groups $(\mathrm{P}=0.79$; Figure 4$)$.

\section{Discussion}

At present, organ shortage is a common problem in the transplantation community. DCD has become the main method for organ donation in China due to its special social environment (13), and the prevalence of obesity in the population has continuously increased the proportion of liver steatosis $(3,4)$. Steatosis and DCD are risk factors of poor prognosis after liver transplantation; thus, steatohepatic donor livers from DCD, especially MS $>30 \%$, are generally considered extremely high risk and are discarded (7). In our study, liver transplantation achieved acceptable results by using moderate steatosis liver from DCD. The short- and long-term survival rates in patients and grafts between the two groups were similar (patient survival: $75 \%$ vs. $85.9 \%, 75 \%$ vs. $78.1 \%, 68.8 \%$ vs. $71.9 \%$, log-rank test, $\mathrm{P}=0.77$; graft survival: $75 \%$ vs. $84.4 \%, 75 \%$ vs. $75 \%, 68.8 \%$ vs. $68.8 \%$, log-rank test, $\mathrm{P}=0.79$ ). We only found a significant difference in the incidence of IPF (11/16 vs. 26/64; $\mathrm{P}=0.02)$. The average peak value of transaminase $\operatorname{AST}(3,469 v s .1,295 \mathrm{P}<0.01)$ was significantly higher in the group with steatosis than in the control group.

Transaminase is a marker of liver cell damage, and TB (total bilirubin) and INR reflect the function of the graft. The results of postoperative laboratory indicators indicate that the MS-DCD group had higher ALT and AST peaks than the NS group. Notably, the steatotic liver is less tolerant to ischemia-reperfusion injury (14), and WIT during DCD increases in ischemia-reperfusion injury. Liver cell damage is more severe in the MS-DCD group. Thus, higher transaminase peaks and IPF incidence rates are obtained. After early symptomatic supportive treatment after surgery, the trend of liver function recovery is nearly the same. The changes in TB and INR imply that the liver function and recovery of the MS-DCD and NS groups are the same. This conclusion has also been confirmed in the application of a severe steatotic donor liver (15).

Decisions on the application of donor liver should fully consider the comprehensive judgment of various factors, including DRI, donor history, acquisition process, and OPO scheduling. In the experience of our center, the importance 
Table 2 Donor characteristics

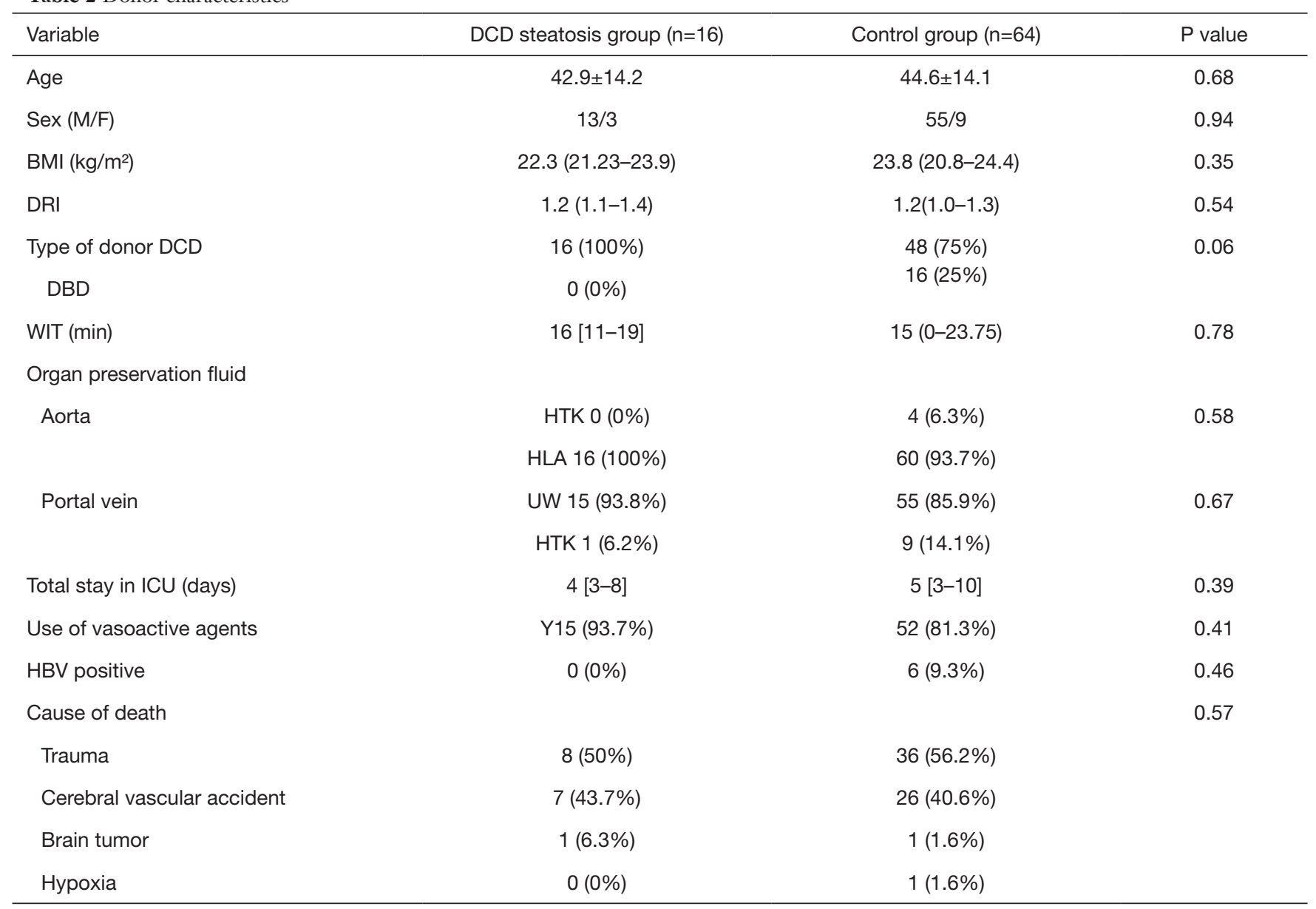

DCD, donation after cardiac death; BMI, body mass index; DRI, donor risk index; DBD, donation after brain death; WIT, warm ischemia time; ICU, intensive care unit; HBV, hepatitis $B$ virus.

of preoperative frozen biopsy should be emphasized. Although studies have shown that computed tomography (CT) or magnetic resonance imaging (MRI) can effectively evaluate the degree of liver steatosis in donors, these methods cannot be completed for donors in most medical environments. Therefore, for DCD livers suspected of steatosis, our center uses preoperative frozen biopsy. This process can be performed after the donor liver is obtained without significantly increasing CIT. Nevertheless, relatively accurate pathological results are used to inform the team's decisions.

Nearly $30 \%$ of donor livers are discarded due to steatotic donor livers (5), and this result has also been observed in our center. The misjudgment in abandonment is unavoidable because of the lack of unified standards. Although the number of 16 samples in this study was relatively small, we hope that the results of this study will increase the understanding of retaining or discarding the DCD steatotic liver and improve organ utilization

High levels of transaminase and IPF can be detected early after DCD steatotic donor liver surgery. Therefore, the levels should be chosen carefully for critically ill patients or patients with high MELD scores. For patients with high risk but relatively low MELD scores during waiting period, such as those with actively progressing liver cancer and repeated active gastrointestinal bleeding, low MELD scores prolong waiting time and make them eventually lose the opportunity for transplantation. In such cases, DCD fatty livers can also be a good choice.

At present, the time of CIT and WIT should be shortened as much as possible in the application strategy of donor livers with moderate steatosis $(16,17)$. Therefore, 
Table 3 Postoperative outcomes and complications

\begin{tabular}{|c|c|c|c|}
\hline Postoperative outcomes & DCD steatosis group $(n=16)$ & Control group $(n=64)$ & $P$ value \\
\hline IPF & $11(68.8 \%)$ & $26(40.6 \%)$ & 0.02 \\
\hline Average peak value of ALT & $901.5(505.3-1,986.8)$ & $594(365.0-1,341.0)$ & 0.20 \\
\hline Average peak value of AST & $3,469(1,693.3-5,360.3)$ & $1,295(720-2,373)$ & $<0.01$ \\
\hline Average peak value of TB & $150(45.2-243.8)$ & $141(67.5-258)$ & 0.81 \\
\hline Overall complication & $11(68.8 \%)$ & $38(59.4 \%)$ & 0.49 \\
\hline Hepatic artery thrombosis & $4(25 \%)$ & $12(18.8 \%)$ & 0.83 \\
\hline Portal vein thrombosis & $3(18.8 \%)$ & $9(14.1 \%)$ & 0.94 \\
\hline \multicolumn{4}{|l|}{ Biliary } \\
\hline Bile leakage & $1(6.3 \%)$ & $2(3.1 \%)$ & 0.49 \\
\hline Biliary stricture & $1(6.3 \%)$ & $7(10.9 \%)$ & 0.93 \\
\hline ICU stay (days) & $8.6(6.6-10.4)$ & $7.8(6.2-12.0)$ & 0.56 \\
\hline
\end{tabular}

DCD, donation after cardiac death; PNF, primary nonfunction; IPF, initial poor function; ALT, alanine transaminase; AST, aspartate transaminase; INR, international normalized ratio; TB, total bilirubin; ICU, intensive care unit.
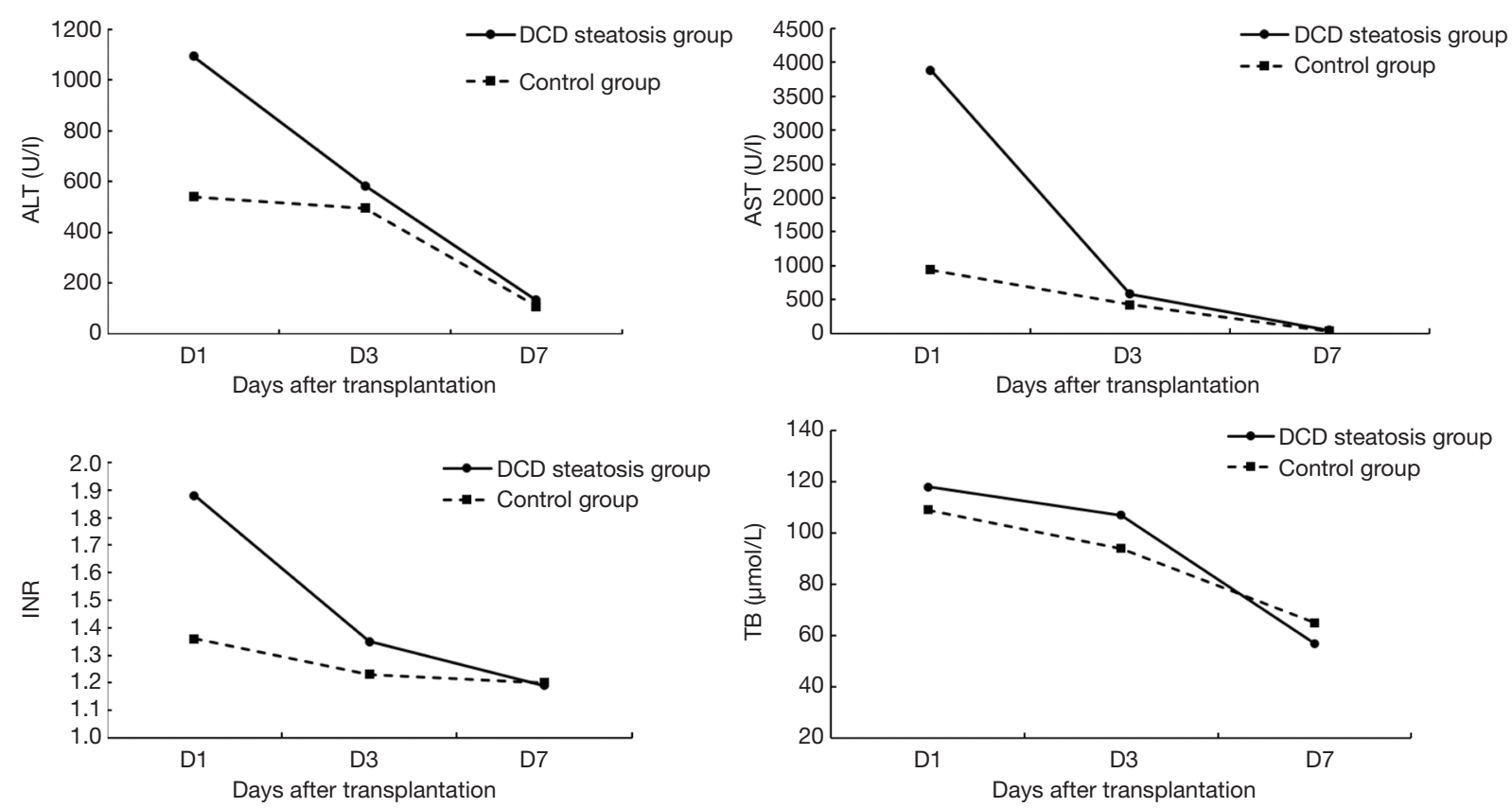

Figure 1 Mean serum levels of ALT (U/I), AST (U/I), INR, and TB ( $\mu \mathrm{mol} / \mathrm{L})$ on post-transplantation days 1, 3, and 7 between the two groups. ALT, alanine transaminase; AST, aspartate transaminase; INR, international normalized ratio; TB, total bilirubin. 

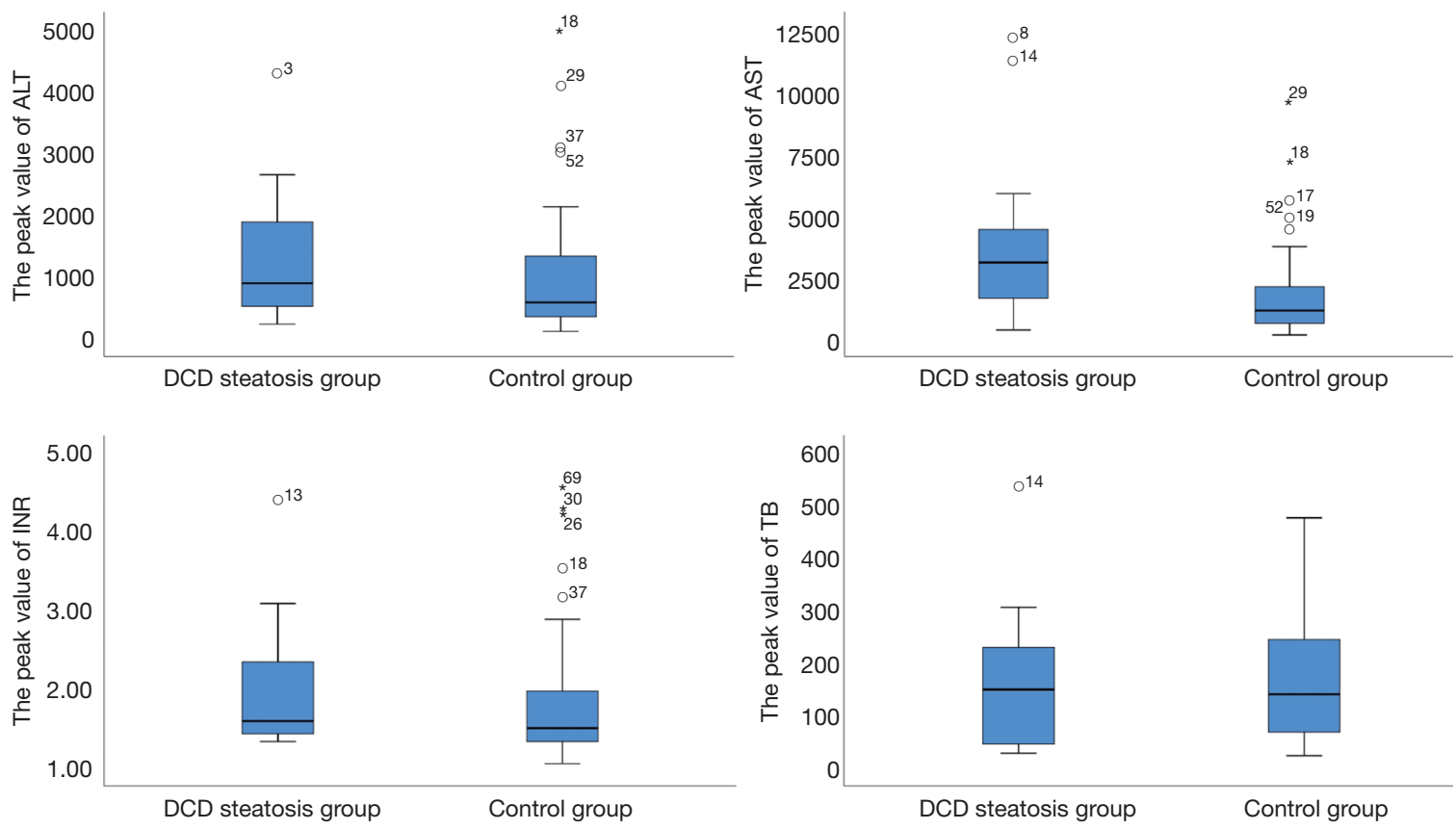

Figure 2 Peak serum levels of ALT (U/I), AST (U/I), INR, and TB ( $\mu$ mol/L) during the week after transplantation between the two groups. ALT, alanine transaminase; AST, aspartate transaminase; INR, international normalized ratio; TB, total bilirubin

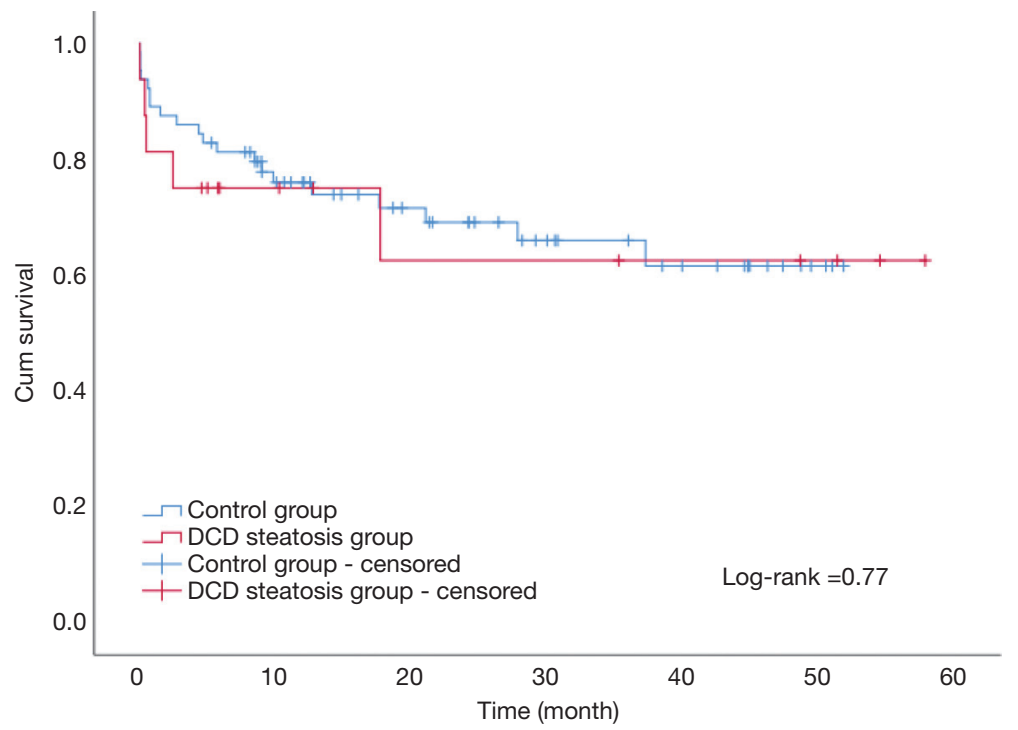

Figure 3 Overall patient survival.

communication and cooperation with the OPO team should be strengthened to optimize the DCD and transport process. However, CIT $<8 \mathrm{~h}$ is often difficult to achieve due to various factors, such as logistics and transportation.
Therefore, advanced organ preservation technologies, like normothermic machine perfusion (18), should be used to shorten the CIT time, allowing for DCD steatotic livers to be utilized. 
Table 4 Comparison of patient and graft survival rates

\begin{tabular}{|c|c|c|c|}
\hline Survival & DCD steatosis group $(n=16)$ & Control group $(n=64)$ & $P$ value \\
\hline 90-day & 75 & 84.4 & 0.61 \\
\hline 1-year & 75 & 75 & $>0.99$ \\
\hline 3-year & 68.8 & 68.8 & $>0.99$ \\
\hline 90-day & 75 & 85.9 & 0.50 \\
\hline 1-year & 75 & 78.1 & $>0.99$ \\
\hline 3-year & 68.8 & 71.9 & $>0.99$ \\
\hline
\end{tabular}

DCD, donation after cardiac death.

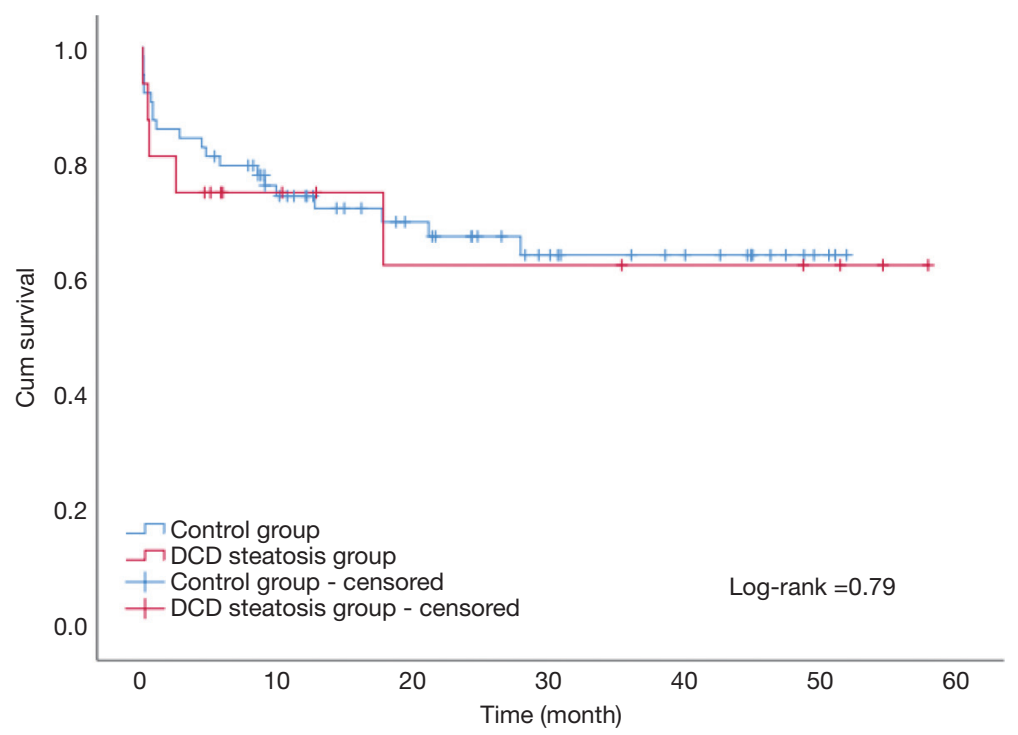

Figure 4 Overall graft survival.

\section{Acknowledgments}

Funding: None.

\section{Footnote}

Reporting Checklist: The authors have completed the STROBE reporting checklist. Available at http://dx.doi. org/10.21037/atm-20-5888

Data Sharing Statement: Available at http://dx.doi. org/10.21037/atm-20-5888

Conflicts of Interest: All authors have completed the ICMJE uniform disclosure form (available at http://dx.doi. org/10.21037/atm-20-5888). The authors have no conflicts of interest to declare.

Ethical Statement: The authors are accountable for all aspects of the work in ensuring that questions related to the accuracy or integrity of any part of the work are appropriately investigated and resolved. The study was conducted in accordance with the Declaration of Helsinki (as revised in 2013). The study was approved by the First Affiliated Hospital, College of Medicine, Zhejiang University (No. 1393-1) and individual consent for this retrospective analysis was waived. 
Open Access Statement: This is an Open Access article distributed in accordance with the Creative Commons Attribution-NonCommercial-NoDerivs 4.0 International License (CC BY-NC-ND 4.0), which permits the noncommercial replication and distribution of the article with the strict proviso that no changes or edits are made and the original work is properly cited (including links to both the formal publication through the relevant DOI and the license). See: https://creativecommons.org/licenses/by-nc-nd/4.0/.

\section{References}

1. Mohamed MA, WN WS, Jaafar J, et al. Regenerated cellulose membrane as bio-template for in-situ growth of visible-light driven C-modified mesoporous titania. Carbohydr Polym 2016;146:166-73.

2. Liu Z, Jia J, Ning H, et al. Systematic Evaluation of the Safety Threshold for Allograft Macrovesicular Steatosis in Cadaveric Liver Transplantation. Front Physiol 2019;10:429.

3. Sherif ZA, Saeed A, Ghavimi S, et al. Global Epidemiology of Nonalcoholic Fatty Liver Disease and Perspectives on US Minority Populations. Dig Dis Sci 2016;61:1214-25.

4. Selzner M, Clavien PA. Fatty liver in liver transplantation and surgery. Semin Liver Dis 2001;21:105-13.

5. Moosburner S, Gassner J, Nosser M, et al. Prevalence of Steatosis Hepatis in the Eurotransplant Region: Impact on Graft Acceptance Rates. HPB Surg 2018;2018:6094936.

6. Andert A, Ulmer TF, Schoning W, et al. Grade of donor liver microvesicular steatosis does not affect the postoperative outcome after liver transplantation. Hepatobiliary Pancreat Dis Int 2017;16:617-23.

7. Spitzer AL, Lao OB, Dick AA, et al. The biopsied donor liver: incorporating macrosteatosis into high-risk donor assessment. Liver Transpl 2010;16:874-84.

8. Dutkowski P, Schlegel A, Slankamenac K, et al. The use of fatty liver grafts in modern allocation systems: risk assessment by the balance of risk (BAR) score. Ann Surg

Cite this article as: Duan X, Yan L, Shen Y, Zhang M, Bai X, Liang T. Outcomes of liver transplantation using moderately steatotic liver from donation after cardiac death (DCD). Ann Transl Med 2020;8(18):1188. doi: 10.21037/atm-20-5888
2012;256:861-8; discussion 868-9.

9. Chen H, Peng CH, Shen BY, et al. Multi-factor analysis of initial poor graft function after orthotopic liver transplantation. Hepatobiliary Pancreat Dis Int 2007;6:141-6.

10. Gabrielli M, Moisan F, Vidal M, et al. Steatotic livers. Can we use them in OLTX? Outcome data from a prospective baseline liver biopsy study. Ann Hepatol 2012;11:891-8.

11. Evrard P, Belgian Working Group on DCDNP. Belgian modified classification of Maastricht for donors after circulatory death. Transplant Proc 2014;46:3138-42.

12. Bruinsma BG, Wu W, Ozer S, et al. Warm ischemic injury is reflected in the release of injury markers during cold preservation of the human liver. PLoS One 2015;10:e123421.

13. Matesanz R, Mahillo B, Alvarez M, et al. Global observatory and database on donation and transplantation: world overview on transplantation activities. Transplant Proc 2009; 41:2297-301.

14. McCormack L, Dutkowski P, El-Badry AM, et al. Liver transplantation using fatty livers: always feasible? J Hepatol 2011;54:1055-62.

15. Wong TC, Fung JY, Chok KS, et al. Excellent outcomes of liver transplantation using severely steatotic grafts from brain-dead donors. Liver Transpl 2016;22:226-36.

16. Frongillo F, Avolio AW, Nure E, et al. Quantification of degree of steatosis in extended criteria donor grafts with standardized histologic techniques: implications for graft survival. Transplant Proc 2009;41:1268-72.

17. El-Badry AM, Breitenstein S, Jochum W, et al. Assessment of hepatic steatosis by expert pathologists: the end of a gold standard. Ann Surg 2009;250:691-7.

18. op den Dries S, Karimian N, Sutton ME, et al. Ex vivo normothermic machine perfusion and viability testing of discarded human donor livers. Am J Transplant 2013;13:1327-35.

(English Language Editor: J. Gray) 